\title{
The Effects of Small Contents of Water on Melt Rheology for Ethylene-Methacrylic Zinc Ionomers
}

\author{
By Akihiro NISHIOKA, ${ }^{1, *}$ Tomonori KODA, ${ }^{2}$ Ken MIYATA,${ }^{3}$ Go MURASAWA ${ }^{4}$ and Kiyohito KOYAMA ${ }^{2}$
}

The effects of small contents of absorbed water on coordinated structure and rheological properties for EMAA-Zn ionomers in terms of dynamic visocoelastic and FT-IR measurement were investigated. To clarify the influence of absorbed water in ionomers, the moisture contents were measured by Karl Fisher Method. It was clarified that zero shear viscosities of EMAAionomers were reduced by small contents of absorbed water. We considered that the drastic change of coordinated structure by little amounts of water might induce acid-cation exchange and affect melt rheological properties such as zero shear viscosities. We consider that absorbed water is one of the key factors to control zero shear viscosity in EMAA-Zn ionomer melts.

KEY WORDS: Ionomer / Absorbed Water / Zero Shear Viscosity / Coodinated Structure /

Ionomers are polymers including a small amount of metal ionic salt groups. Poly(ethylene-co-methacrylic acid)(EMAA) neutralized with Zinc metal cations is widely used for industrial applications such as golf ball, film for food package and so on. The structure and mechanical properties of ionomers in both solid and melt states were extensively investigated. Ionic groups tend to form ionic aggregates in the hydrophobic polymer matrix. ${ }^{1-5}$ Dielectric measuerment, dynamic mechanical relaxation and melt rheological studies show that a microphase separation of ionic aggregates and the hydrocarbon matrix takes place depending on the neutralization degree. For solid state, Hirasawa et al. and Tachino et al. suggest that ionic aggregates are formed at a degree of neutralization larger than $80 \%$ in EMAA-Zn ionomer. ${ }^{6,7}$ For melt state, Nishioka et al. have already reported rheological investigation showing that ionic aggregates are formed at neutralization degree of $40 \%{ }^{8}$ Their results show that in case of EMAA-Zn ionomers, zero shear visicosities tend to increase by formation of ionic aggregates above neutralization of $40 \%$. In general, especially for EMAA-Zn ionomers, one of the disadvantage is that melt viscosity is too high in the range of high neutralization degree.

Some melt rheological studies on EMAA ionomers have been published to examine the role of ion aggregates at molten stage. ${ }^{9-13}$ There are some factors, which can reduce viscosity of EMAA-Zn ionomers, such as adding low molecular weight acid, absorbed water and so on. Several studies have been carried out about reducing melt viscosity of EMAA ionomers with keeping the constant neutralization degree by blending additives. Iwami et al. demonstrated that viscosity of EMAA ionomers can be reduced by blending static acid. ${ }^{14}$ Nishioka et al. reported that, by blending low molecular weight acid(motanic acid: $\mathrm{C}_{27} \mathrm{H}_{55} \mathrm{COOH}$ ), visocosity of EMAA- $\mathrm{Zn}$ ionomer can be reduced caused by inducing newly coordina- tion structure. ${ }^{15}$ This change in coordination structure induces "acid-cation exchange mechanism" proposed by Vanhoore and Register. ${ }^{13}$ We consider that one of the key factors to reduce viscosity is controlling coordinate structure of EMAA-Zn ionomers such as tetra or hexa-coordinate structures.

Many researchers reported that coordinate structure for EMAA-Zn ionomers were strongly affected by absorbed water. ${ }^{16-20}$ We consider that controlling rheological properties such as viscosity for EMAA-Zn ionomers is one of the important research subject in the field of industry applications. With this industrial importance, the purpose of this study is to estimate the effect of absorbed water on melt rheological properties of EMAA-Zn ionomers in terms of dynamic viscoelastic and FT-IR measurements.

\section{SAMPLE AND EXPERIMENTAL}

Poly-(ethylene-co-methacrylic acid) (EMAA, $M_{\mathrm{n}}=19,200$, $\left.M_{\mathrm{w}}=94,500\right)$, whose methacrylic acid content was $15.0 \mathrm{wt} \%$ $(5.4 \mathrm{~mol} \%)$, was used as the matrix of ionomers. Ionomers used in this study were EMAA neutralized partially by Zinc( $\mathrm{Zn})$ ions. These are designated as EMAA-xxZn. Here, $\mathrm{xx}$ means value of neutralization degree of ionomers. All these neutralized samples and EMAA were kindly provided by Mitsui-DuPont Polychemicals Co. Ltd, Japan. These EMAA$\mathrm{Zn}$ ionomers were neutralized by $\mathrm{ZnO}$ (Zinc Oxide) blended with EMAA in melt state. To clarify the influence of absorbed water in ionomers, before experiments, a great attention was paid for the moisture content. It sensitively affects melt rheological properties.

Two kinds of drying conditions were chosen, which were $60^{\circ} \mathrm{C}$ for $3 \mathrm{~d}$ and $80^{\circ} \mathrm{C}$ for $14 \mathrm{~d}$, respectively. All pellet samples were dried in vacuum oven under two different

\footnotetext{
${ }^{1}$ Cooperative Research Center (CRC), Yamagata University

2Department of Polymer Science and Enginieering, Yamagata University

${ }^{3}$ Venture Business Laboratory (VBL), Yamagata University

${ }^{4}$ Department of Mechanical Engineering, Yamagata University

*To whom correspondence should be addressed (Tel/Fax: +81-238-26-3207, E-mail: nishioka@yz.yamagata-u.ac.jp).
} 
Table I. The value of water contents under different drying conditions

\begin{tabular}{lcccc}
\hline & Zn59\% & Zn40\% & Zn20\% & E/MAA \\
\hline Before drying & $1510 \mathrm{ppm}$ & $1250 \mathrm{ppm}$ & $870 \mathrm{ppm}$ & $220 \mathrm{ppm}$ \\
\hline $60^{\circ} \mathrm{C}$ 3 d & $750 \mathrm{ppm}$ & $330 \mathrm{ppm}$ & $210 \mathrm{ppm}$ & $70 \mathrm{ppm}$ \\
\hline $80^{\circ} \mathrm{C} 14 \mathrm{~d}$ & $220 \mathrm{ppm}$ & $160 \mathrm{ppm}$ & $140 \mathrm{ppm}$ & $80 \mathrm{ppm}$ \\
\hline
\end{tabular}

conditions mentioned above. The water percentages of EMAA, EMAA-Zn under different dry conditions were measured by Karl-Fischer method (METTLER KF-Coulometer DL37, Drying oven DO337) at $180^{\circ} \mathrm{C}$ for $30 \mathrm{~min}$ under nitrogen $(99.999 \%)$ flow $(200 \mathrm{~mL} / \mathrm{min})$, and value of water contents before and after drying in samples were listed in Table I.

About $10-\mu \mathrm{m}$-thick films for fourier transform infrared spectroscopic (FT-IR) analysis were prepared by compression molding at $453 \mathrm{~K}$. IR spectra were measured using a Nicolet Model Magna-IR Spectrometer 750 at room temperature, where 32 scans at a resolution of $4 \mathrm{~cm}^{-1}$ were signal averaged.

Experiments for oscillatory shear were conducted using a rotational rheometer (Rheometrics model: ARES) under nitrogen atmosphere. The geometries of the test fixtures were parallel disks with a diameter of $25 \mathrm{~mm}$ for all samples. The input strain in oscillatory shear was sufficiently small to provide reliable linear viscoelastic data. The measurement temperatures were $140,160,180,200,220^{\circ} \mathrm{C}$ for EMAA and EMAA-Zn ionomers, where all samples showed the excellent thermal stability and reproducibility. All disk shaped samples for measurements were produced by hot press method (Toyoseiki, Co. Ltd, Model: Mini-Test Press-10) at $130^{\circ} \mathrm{C}$ for EMAA and $190^{\circ} \mathrm{C}$ for EMAA-Zn ionomers.

\section{RESULTS AND DISCUSSION}

Oscillatory shear measurements for EMAA and its ionomers neutralized by $\mathrm{Zn}$ metal cations in different dry conditions were performed. These measurements were carried out at $140^{\circ} \mathrm{C}$, $160^{\circ} \mathrm{C}, 180^{\circ} \mathrm{C}, 200^{\circ} \mathrm{C}$ and $220^{\circ} \mathrm{C}$, which are substantially above their melting temperature. The resulting storage modulus $\mathrm{G}^{\prime}$ and loss modulus $\mathrm{G}^{\prime \prime}$ curves were horizontally shifted to the curve at $140{ }^{\circ} \mathrm{C}$ as reference temperature in order to obtain their master curves. Figure 1 shows the master curves of EMAA59Zn under different dry conditions. Time-temperature super position is applicable for all samples in all measurement conditions. From these results, zero shear viscosity $\eta_{0}$ at $140{ }^{\circ} \mathrm{C}$ can be obtained from the value in sufficiently low frequency region of the master curves.

Figure 2 shows zero shear viscosity $\eta_{0}$ of EMAA-Zn ionomers as a function of neutralization degree with log-linear plots for two different dry conditions. For both samples, zero shear viscosity $\eta_{0}$ firstly rises linearly until neutralization degree reaches to about $40 \%$. Below neutralization degree of $40 \%$, tendencies of $\eta_{0}$ were independent on drying conditions. Above neutralization degree of $40 \%$, tendencies of $\eta_{0}$ were remarkably different between the two dry conditions. In case of dry condition at $80^{\circ} \mathrm{C}$ for $14 \mathrm{~d}, \eta_{0}$ starts to deviating from linearity at neutralization degree of $40 \%$. On the other hand, $\eta_{0}$

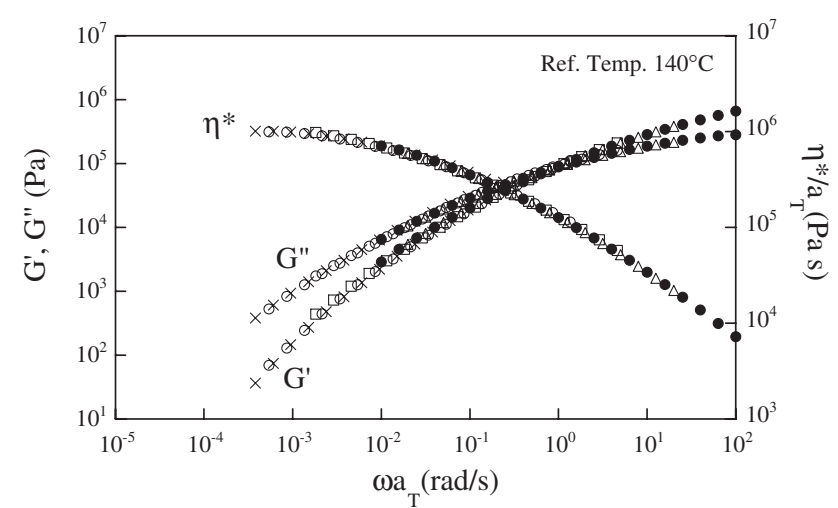

(a) $60^{\circ} \mathrm{C} 3 \mathrm{~d}$

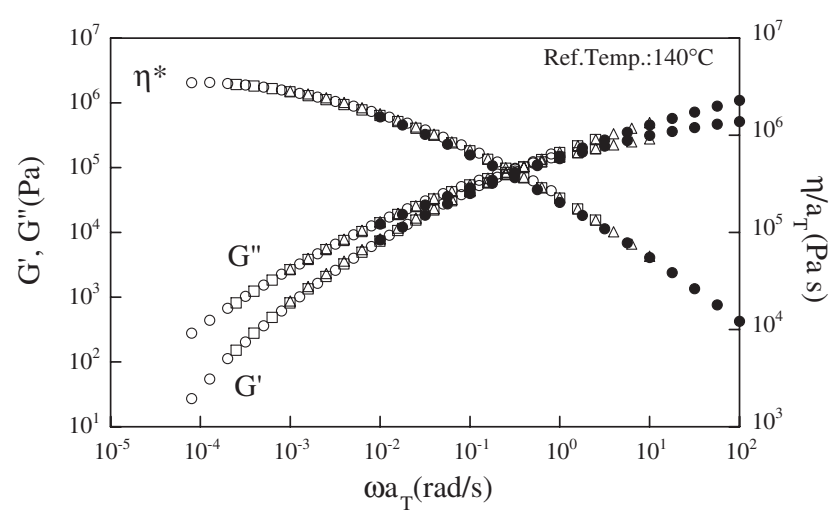

(b) $80^{\circ} \mathrm{C} 14 \mathrm{~d}$

Figure 1. Master curve of $\mathrm{G}^{\prime}, \mathrm{G}^{\prime \prime}$ and $\eta^{*}$ for EMAA-59Zn $\left(140^{\circ} \mathrm{C}:-160^{\circ} \mathrm{C}\right.$ : $\left.\triangle, 180^{\circ} \mathrm{C}: \square, 200^{\circ} \mathrm{C}: \bigcirc, 220^{\circ} \mathrm{C}: \mathbf{x}\right)$. The reference temperature is $140^{\circ} \mathrm{C}(\mathrm{O})$

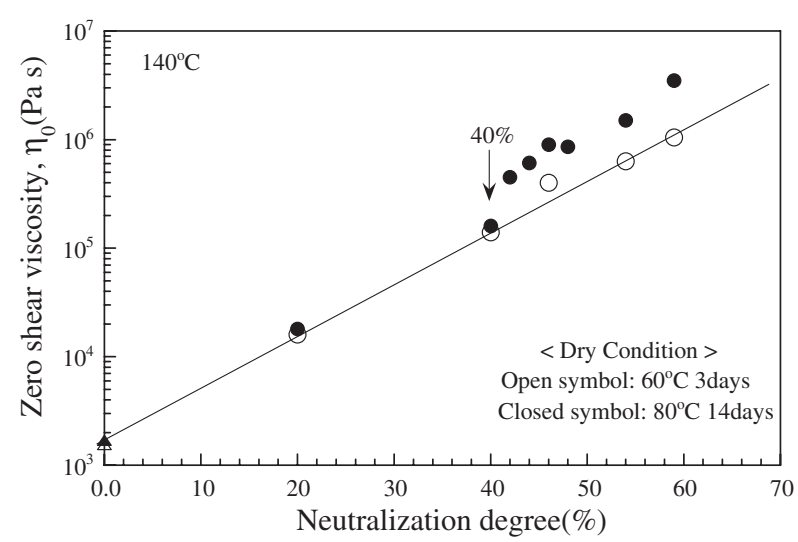

Figure 2. Zero shear viscosities $\eta_{0}$ as a function of neutralization degree for EMAA-Zn ionomers.

keeps linearly above $40 \%$ for dry condition at $60^{\circ} \mathrm{C}$ for $3 \mathrm{~d}$. According to these results, it is easy to understand that this difference of tendency for $\eta_{0}$ above $40 \%$ is come from degree of water content in EMAA-Zn ionomers.

The reason why the effect of water contents on $\eta_{0}$ appeared for only above $40 \%$ shall be discussed. We consider that the cause of reduction for $\eta_{0}$ by degree of water contents in EMAA-Zn ionomers can be explained by "acid-cation ex- 
change mechanism" proposed by Vanhoorne and Register. ${ }^{13}$ They studied the effect of the protons of acid groups on the melt rheology of partially neutralized EMAA-Zn ionomer. By removing the protons by etherification of the acid group, they have found that the role of the protons in sodium ionomers is different from that in zinc ionomers. ${ }^{13}$ In case of sodium ionomers, a sodium ion is not only paired with a single $\mathrm{COO}^{-}$ but also with two $\mathrm{COOH}$ acid groups by coordinate bonds. Due to this environment, $\mathrm{COOH}$ acid groups tend to exist around sodium ions, and therefore the sodium ion and a proton can be exchanged between $\mathrm{COO}-\mathrm{Na}$ and $\mathrm{COOH}$. Whereas, EMAA-Zn ionomer has tetra-coordinated structure, in which a zinc ion is coordinated with two $\mathrm{COO}^{-}$groups due to ionic bonds, and no $\mathrm{COOH}$ group exists around zinc ions. In this situation, a zinc ion has no possibility to receive the effect of proton from free carboxylic acid. They concluded that, in zinc ionomers, the acid-cation exchange does not take place.

We consider that a drastic change of coordinated structure by little amounts of water also may induce acid-cation exchange and affect melt rheological properties such as zero shear viscosities. Our recent data shows that incorporation of low molecular acid(montanic acid: $\mathrm{C}_{27} \mathrm{H}_{55} \mathrm{COOH}$ ) decreases zero shear viscosity of EMAA-Zn ionomers. These results is explained by coordinate structural change detected by FT-IR ${ }^{15}$ and can be related to the local environmental change for the present case which is derived by the incorporation of small contents of water. We hence consider coordinated structure change, in ionic aggregates resulted in a remarkable reduction of zero shear viscosity.

To clarify the effect of absorbed water on coordinated structure of EMAA-Zn ionomers, we carried out FT-IR measurements. Figure 3 shows results of FT-IR under two different dry conditions. Apparent change took place only in the range between 1500 and $1650 \mathrm{~cm}^{-1}$. EMAA-59Zn under dry condition at $80^{\circ} \mathrm{C}$ for $14 \mathrm{~d}$ (Figure 1(a)) gave a characteristic peak, corresponding to the carboxylate asymme$\operatorname{tric}\left(v_{\mathrm{a}}(\mathrm{COO}-)\right)$ stretching mode at $1585 \mathrm{~cm}^{-1}$, which means tetracoordinate zinc carboxylate structure. On the other hand,

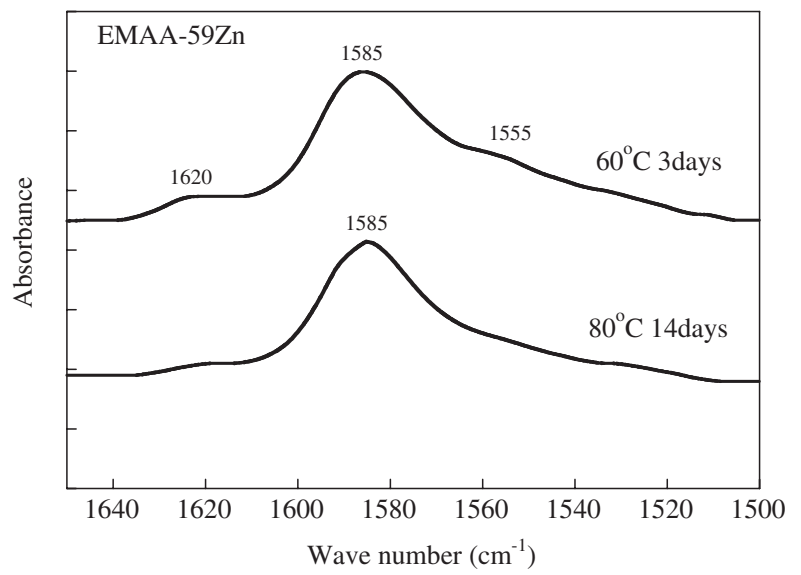

Figure 3. Infrared spectra in the range of $1500-1650 \mathrm{~cm}^{-1}$ for EMAA-59Zn at room temperature. unexpected striking changes was observed for dry condition $60{ }^{\circ} \mathrm{C}$ for $3 \mathrm{~d}$. Those are appearance of characteristic band of $1555 \mathrm{~cm}^{-1}$, and increase of the band of $1620 \mathrm{~cm}^{-1}$. The band of $1555 \mathrm{~cm}^{-1}$ appearing as a weak shoulder can be combination of two bands of 1536 and $1565 \mathrm{~cm}^{-1}$ which are assigned to hexacoordinated zinc carboxylate. ${ }^{18}$ Reinforced peak of $1620 \mathrm{~cm}^{-1}$ corresponds to an acid salt structure. ${ }^{18}$ These effects due to small amount of water in feature of FT-IR peaks support the conclusion that difference in rheology comes from change in molecular coordination structure.

\section{CONCLUSIONS}

In this paper, we focus on how structure change induced by absorbed water affects the rheological property. The present study on the effects of small contents of water on melt rheology for EMAA-Zn ionomers provided the following new findings. (1) Dynamic shear properties of EMAA-Zn ionomers under different dry conditions shows that the time-temperature superposition principle is applicable for all samples from $140{ }^{\circ} \mathrm{C}$ to $220^{\circ} \mathrm{C}$. The zero shear viscosity $\eta_{0}$ can be obtained from the value in sufficiently low frequency region of master curve for all samples.

(2) The values of zero shear viscositie $\eta_{0}$ are different depending on dry conditions. This change in $\eta_{0}$ comes from whether the acid cation exchange occurs or not by absorbed water under different dry conditions. Considering FT-IR results for EMAA-Zn ionomers, coordinate structure change of EMAA-Zn was led by absorbed water. This coordinate structure change induce "acid cation exchange mechanism" and apparent reduction of $\eta_{0}$ in the range of high neutralization degree $(>40 \%)$ was observed. We conclude that absorbed water in EMAA-Zn ionomer is one of novel factors to control rheological properties.

Acknowledgment. We are grateful to Mitsui-DuPont Polychemicals Co., Ltd for providing samples and helpful discussions. The authors would also like to thank Prof. Dr. K. Osaki (Kyoto University, Japan), Prof. Dr. Kutsumizu for their valuable comments. This work was supported by Grants-in-Aid from the Ministry of Education, Culture, Sports, Science and Technology of Japan, No. 19710075 and No. 18068003 (PriorityArea "Soft Matter Physics").

Received: October 3, 2007

Accepted: January 16, 2008 Published: February 26, 2008

\footnotetext{
REFERENCES

1. A. Eisenberg, Macromolecules, 3, 147 (1970).

2. R. Longworth and D. J. Vaughan, Nature, 218, 85 (1968).

3. D. Yarusso and S. L. Cooper, Macromolecules, 16, 1871 (1983).

4. D. Yarusso and S. L. Cooper, Polymer, 16, 371 (1985).

5. W. J. Macknight, W. P. Taggart, and R. S. Stein, J. Polym. Sci. Symp., 45 (1974).

6. J. Janzen and R. H. Colby, J. Mol. Struct., 485-486, 569 (1999).

7. F. Tanaka and S. F. Edwards, Macromolecules, 25, 1516 (1992).
} 
8. A. Nishioka, M. Nishio, T. Koda, S. Ikeda, and K. Koyama, Nihon Reoroji Gakkaishi, 33, 201 (2005).

9. K. Sakamoto, W. J. MacKnight, and R. S. Porter, J. Polym. Sci. Part 2, 8, 277 (1970).

10. T. R. Earnest, Jr. and W. J. MacKnight, J. Polym. Sci., Polym. Phys., Ed., 16, 143 (1978).

11. W. J. MacKnight and T. R. Earnest, Jr., J. Polym. Sci., Macromol. Rev., 16, 41 (1981).

12. M. R. Tant and G. L. Wilkes, J. Macromol. Sci. Rev., C28, 1 (1988).

13. P. Vanhoorne and R. A. Register, Macromolecules, 29, 598 (1996).

14. I. Iwami, Y. Kawasaki, and H. Kinoshita, Kokoku, Patent No. 013726,
(1981).

15. A. Nishioka, T. Takahashi, Y. Masubuchi, J. Takimoto, and K. Koyama, Polymer, 42, 7907 (2001).

16. B. P. Grady, J. A. Floyd, W. B. Genetti, P. Vanhoorne, and R. A. Register, Polymer, 40, 283 (1999).

17. T. Ishioka, Polym. J., 25, 1147 (1993).

18. B. P. Grady, Macromolucules, 32, 2983 (1999).

19. A. Welty, S. Ooi, and B. P. Grady, Macromolecules, 32, 2989 (1999).

20. M. M. Coleman, J. Y. Lee, and P. C. Painter, Macromolecules, 23, 2339 (1990). 